Polarization-induced hole doping in N-polar III-nitride LED grown by metalorganic chemical vapor deposition

Long Yan, Yuantao Zhang, Xu Han, Gaoqiang Deng, Pengchong Li, Ye Yu, Liang Chen, Xiaohang Li, and Junfeng Song

Citation: Appl. Phys. Lett. 112, 182104 (2018); doi: 10.1063/1.5023521

View online: https://doi.org/10.1063/1.5023521

View Table of Contents: http://aip.scitation.org/toc/apl/112/18

Published by the American Institute of Physics

PHYSICS TODAY

MANACER'S GUIDE

WHITEPAPERS
READ NOW

PRESENTED BY

Accelerate R\&D with Multiphysics Simulation 


\title{
Polarization-induced hole doping in N-polar III-nitride LED grown by metalorganic chemical vapor deposition
}

\author{
Long Yan, ${ }^{1}$ Yuantao Zhang, ${ }^{1, a)}$ Xu Han, ${ }^{1}$ Gaoqiang Deng, ${ }^{1}$ Pengchong Li, ${ }^{1}$ Ye Yu, ${ }^{1}$ \\ Liang Chen, ${ }^{1}$ Xiaohang Li, ${ }^{2}$ and Junfeng Song ${ }^{1}$ \\ ${ }^{1}$ State Key Laboratory of Integrated Optoelectronics, College of Electronic Science and Engineering, \\ Jilin University, Qianjin Street 2699, Changchun 130012, China \\ ${ }^{2}$ King Abdullah University of Science and Technology (KAUST), Advanced Semiconductor Laboratory, \\ Thuwal 23955-6900, Saudi Arabia
}

(Received 25 January 2018; accepted 23 April 2018; published online 3 May 2018)

\begin{abstract}
Polarization-induced doping has been shown to be effective for wide-bandgap III-nitrides. In this work, we demonstrated a significantly enhanced hole concentration via linearly grading an N-polar $\mathrm{Al}_{\mathrm{x}} \mathrm{Ga}_{1-\mathrm{x}} \mathrm{N}(\mathrm{x}=0-0.3)$ layer grown by metal-organic chemical vapor deposition. The hole concentration increased by $\sim 17$ times compared to that of N-polar p-GaN at $300 \mathrm{~K}$. The fitting results of temperature-dependent hole concentration indicated that the holes in the graded $\mathrm{p}$-AlGaN layer comprised both polarization-induced and thermally activated ones. By optimizing the growth conditions, the hole concentration was further increased to $9.0 \times 10^{17} \mathrm{~cm}^{-3}$ in the graded AlGaN layer. The N-polar blue-violet light-emitting device with the graded p-AlGaN shows stronger electroluminescence than the one with the conventional p-GaN. The study indicates the potential of the polarization doping technique in high-performance N-polar light-emitting devices. Published by AIP Publishing. https://doi.org/10.1063/1.5023521
\end{abstract}

GaN and AlN and their ternary alloys have a direct bandgap ranging from $3.4(\mathrm{GaN})$ to $6.2 \mathrm{eV}$ (AlN). Because of their large bandgap, they are widely used in the fabrication of ultraviolet (UV) devices such as UV light-emitting diodes (LEDs), laser diodes, and UV solar blind detectors. ${ }^{1-3}$ However, the low conductivity in the p-type AlGaN layer has hindered the development of high efficiency and output power devices largely due to the high activation energy of the $\mathrm{Mg}$ acceptor, which is $\sim 150 \mathrm{meV}$ in $\mathrm{GaN}$ and increases monotonically with the $\mathrm{Al}$ content in AlGaN. ${ }^{4}$ This means a very small percentage of acceptor ionization (less than $0.5 \%$ ) in $\mathrm{AlGaN}$ at room temperature.

Recently, polarization-induced hole doping has been demonstrated as a feasible approach to increasing the ionization probability of the $\mathrm{Mg}$ acceptor. Negative bound unbalanced charges can be created in a compositionally graded AlGaN layer because of different magnitudes of the polarization in $\mathrm{AlGaN}$ with different $\mathrm{Al}$ contents. Then the holes are field-ionized from the $\mathrm{Mg}$ acceptors to neutralize the negative bound polarization charges. The holes generated by this method have a high density with a weak temperature dependence. ${ }^{5}$ Up to now, this method has been used to realize p-type doping in metal- and N-polar AlGaN by molecular beam epitaxy (MBE) and metal-polar AlGaN by metalorganic chemical vapor deposition (MOCVD). ${ }^{5-7}$ The $\mathrm{N}$-polar GaN-based structures grown by MOCVD have typically high background electron concentration. ${ }^{8}$ Few works have been reported on achieving p-type conduction in $\mathrm{N}$-polar AlGaN by MOCVD. In our previous work, ${ }^{9}$ we reduced the background electron concentration of N-polar GaN by optimizing the V/III ratio. Based on this optimization, we achieved N-polar p-type AlGaN by using the

a)E-mail: zhangyt@jlu.edu.cn polarization-induced doping method with the MOCVD technique. Moreover, in previous works on polarization doping, the measured hole concentrations always show a slight rise with increasing temperature, but the authors failed to offer any clear explanation. In this work, we proposed a model to explain the difference between the simulated polarization charge density and the measured hole concentration.

The samples used in this study were grown on sapphire substrates by using an AIXTRON CCS $3 \times 2^{\prime \prime}$ FT MOCVD system. Triethylgallium (TEGa), trimethylgallium (TMGa), trimethylaluminum (TMAl), and high-purity ammonia $\left(\mathrm{NH}_{3}\right)$ were used as the source precursors for $\mathrm{Ga}, \mathrm{Al}$, and $\mathrm{N}$, respectively. Bis-cyclopentadienyl magnesium $\left(\mathrm{Cp}_{2} \mathrm{Mg}\right)$ was employed as the p-type dopant. First, a 2 - $\mu$ m-thick N-polar GaN template was initially grown on sapphire substrates, and the detailed growth conditions can be found in our previous study. ${ }^{10}$ Then, an 75 -nm-thick Mg-doped AlGaN layer with the $\mathrm{Al}$ content linearly graded from 0 to 0.3 was grown on the template at $1020^{\circ} \mathrm{C}$. To realize the graded $\mathrm{Al}$ content, the TMAl flow rate was increased linearly from 0 to $15 \mu \mathrm{mol} / \mathrm{min}$, and the TMGa flow rate was decreased linearly from 50 to $35 \mu \mathrm{mol} / \mathrm{min}$ during the growth process. Meanwhile, the $\mathrm{Cp}_{2} \mathrm{Mg}$ and $\mathrm{NH}_{3}$ flow rates were kept at $60 \mathrm{nmol} / \mathrm{min}$ and $2.2 \times 10^{5} \mu \mathrm{mol} / \mathrm{min}$, respectively. Finally, the as-grown sample was annealed at $720^{\circ} \mathrm{C}$ in a nitrogen ambient to activate the $\mathrm{Mg}$ acceptor. For comparison, we prepared another reference sample, where a 100-nm-thick Mg-doped GaN layer with the same growth conditions of the $\mathrm{Mg}$-doped AlGaN layer was grown on the $2-\mu$ m-thick $\mathrm{N}$-polar GaN template as we mentioned above. The Al content and strain were examined using an X-ray diffractometer (XRD; Rigaku Ultima IV). The electrical properties were investigated by Hall effect measurements (ACCENT HL5500PC, UK). The Ohmic contacts were realized by 
depositing Ni/Au metal layers using a rapid thermal annealing process.

To check the $\mathrm{Al}$ content distribution, the XRD 20- $\omega$ scan was conducted and simulated around the $(10 \overline{1} 5)$ peak of $\mathrm{GaN}$ and $\mathrm{AlGaN}$, as shown in Fig. 1(a). The stronger peak located in $105.5^{\circ}$ belongs to the GaN template. The broad and flat shoulder peak near the GaN peak is from $\mathrm{AlGaN}$, which means that the linearly graded distribution of the $\mathrm{Al}$ content in the AlGaN layer is formed. The close match between the measured and the simulated data indicates that the component and the thickness of the graded AlGaN layer can be accurately controlled by the MOCVD process. To further confirm the linear distribution of the $\mathrm{Al}$ content in the AlGaN layer, XRD reciprocal space mapping (RSM) measurements were performed and the results are shown in Fig. 1(b). According to the tail profile above the reciprocal space lattice point of $\mathrm{GaN}$, we can confirm that the $\mathrm{Al}$ content has a linear distribution in $\mathrm{AlGaN}$, which is consistent with the $2 \theta-\omega$ scanning measurement result. At the same time, the overlap between the tail profile and the fully strained line (the dotted pink line) means that there is no tensile strain relaxation in the graded AlGaN layer. When AlGaN is in the tensile strain state, the direction of piezoelectric polarization is the same as that of spontaneous polarization. ${ }^{11}$ In the fully strained graded $\mathrm{AlGaN}$ layer, the magnitude of piezoelectric polarization increases with the increasing $\mathrm{Al}$ content, which
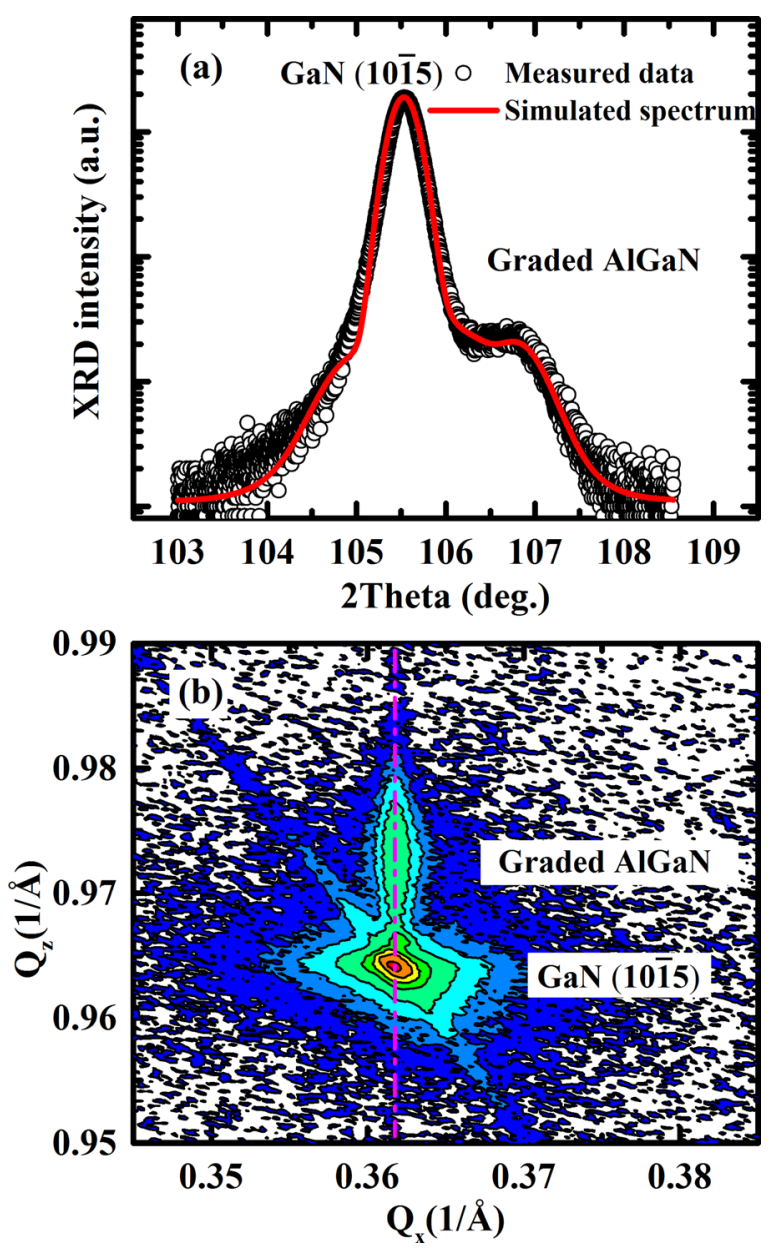

FIG. 1. X-ray diffraction spectrum (a) and reciprocal space map (b) of the (1015) reflection plane for the graded $\mathrm{AlGaN}$ on the $\mathrm{GaN}$ template. is the same as the trend of spontaneous polarization. Therefore, the fully strained state in the graded AlGaN layer can further increase the polarization gradient.

Based on the distribution of the $\mathrm{Al}$ content and the stress state in the graded $\mathrm{AlGaN}$, we can estimate the density of polarization charge, which is created by the difference in polarization in the graded AlGaN, through the formula $\rho_{\pi}(\mathrm{z})=\nabla P(z)$, where $\nabla$ is the divergence operator and $P(z)$ is the sum of spontaneous and piezoelectric polarizations. If the $\mathrm{Al}$ content is small enough, a simple linear model may be used to calculate the polarization charge density of $\mathrm{AlGaN},{ }^{5}$ which is given by $5 \times 10^{13} \times(x / d) \mathrm{cm}^{-3}$, where $x$ is the $\mathrm{Al}$ content at the ended interference of the graded $\mathrm{AlGaN}$ and $d$ is the thickness of the graded layer. Therefore, the density of the polarization charges in our graded AlGaN layer may be as high as $2 \times 10^{18} \mathrm{~cm}^{-3}$. In theory, the fieldionized free holes have the same concentration with the polarization charge.

To probe the hole concentration and verify the acceptoractivation method, temperature dependent Hall measurements were carried out on both the graded $\mathrm{p}-\mathrm{AlGaN}$ and the reference sample $(\mathrm{p}-\mathrm{GaN})$. The results are shown in Fig. 2(a). The hole concentration of the graded $\mathrm{p}-\mathrm{AlGaN}$ layer is $6.4 \times 10^{17} \mathrm{~cm}^{-3}$ at $300 \mathrm{~K}$, which is $\sim 17$ times higher than that of the p-GaN layer $\left(3.7 \times 10^{16} \mathrm{~cm}^{-3}\right)$. As the temperature decreases from 450 to $150 \mathrm{~K}$, the hole concentration in the $\mathrm{p}-\mathrm{GaN}$ layer (the green triangles) decreases exponentially, which is the typical freezeout phenomenon for thermally activated holes. However, the hole concentration of the graded AlGaN layer (the blue squares) shows a weak temperature dependence. The absence of freezeout in the graded AlGaN layer reveals that the holes in the graded AlGaN layer are mainly created by the polarization electric field. Besides, it is worth noting that the measured hole concentration of the graded AlGaN layer is lower than the density of polarization charge (the gray solid line). Meanwhile, when the temperature is higher than $250 \mathrm{~K}$, the hole concentration shows a slight rise with increasing temperature. This behavior is similar to the freezeout phenomenon from thermally activated holes. Hence, we speculate that the holes in the graded AlGaN layer include both field-ionized holes and thermally activated holes. When the temperature is low
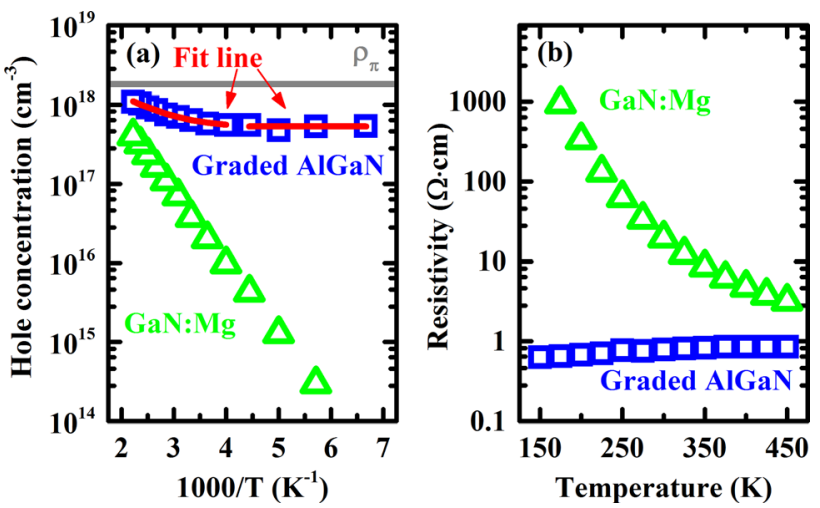

FIG. 2. Temperature dependent hole concentration (a) and resistivity (b) of the graded p-AlGaN and the p-GaN. The gray line indicates the concentration of polarization-induced volume charges predicted by the theory. The red line is the fitting result using Eq. (1). 
enough, such as $150 \mathrm{~K}$ to $225 \mathrm{~K}$, the thermally activated holes are relatively sparse. The measured hole concentration of the graded AlGaN layer is similar to the concentration of field-ionized holes, which is temperature-independent. When the temperature further goes up, the thermally activated hole concentration gets close to the field-ionized hole concentration. As a consequence, the measured hole concentration is affected by the thermally activated hole, which is proportional to the temperature. In this situation, the concentration of the measured holes can be expressed by

$$
p=p_{0}+p_{\pi}=N_{A}-N_{D}-p_{A},
$$

where $p_{0}$ is the concentration of thermally activated holes, $p_{\pi}$ is the concentration of field-ionized holes which is equal to the density of polarization charge, $N_{A}$ is the acceptor concentration, $N_{D}$ is the compensating donor concentration, and $p_{A}$ is the inactivated acceptor concentration. To verify this inference, the measured hole-concentration data are fitted in two different temperature ranges. When the temperature is lower than $225 \mathrm{~K}$, we only consider the field-ionized holes. However, when the temperature is higher than $250 \mathrm{~K}$, the temperature-dependent hole concentrations are fitted by Eq. (2) as follows, which combines Eq. (1) with the singleacceptor model and Boltzmann statistics:

$$
\frac{p_{0}\left(p_{0}+\rho_{\pi}+N_{D}\right)}{N_{A}-N_{D}-p_{0}-\rho_{\pi}}=\frac{N_{V}}{g} \exp \left(-\frac{E_{A}}{k_{B} T}\right),
$$

where $N_{V}$ is the effective density of states in the valence band $\left[2\left(2 \pi m_{h}{ }^{*} k_{B} T\right)^{3 / 2} / h^{3}\right], g$ is the acceptor degeneracy factor ( 4 for GaN), ${ }^{12} E_{A}$ is the acceptor activation energy, $k_{B}$ is the Boltzmann constant, and $T$ is the absolute temperature. The good agreement between the model predictions (the red solid lines) and the measured hole concentrations in the entire temperature range verifies the assumed model. However, according to the fitted results, the polarization charge density in our samples is only $\sim 30 \%$ of the calculated one. We speculate that it can be attributed to the screening effect caused by defects. However, further investigations are needed to demonstrate it. Besides, temperature dependent resistivities of the p-GaN layer and the graded p-AlGaN layer were also measured, and the results are shown in Fig. 2(b). The resistivity of the graded AlGaN layer is $\sim 0.8 \Omega \mathrm{cm}$ at $300 \mathrm{~K}$, which is far less than that of the p-GaN layer $(\sim 19.6 \Omega \mathrm{cm})$. As the temperature increases from $150 \mathrm{~K}$ to $450 \mathrm{~K}$, the resistivity of the $\mathrm{p}-\mathrm{GaN}$ layer decreases by more than two orders of magnitude. In contrast, the resistivity of the graded p-AlGaN layer shows a slow increase with increasing temperature. Our result is very similar to that reported in Ref. 5 , in which the authors attributed this metal-like behavior to the absence of hole freezeout and the decrease in mobility with the increase in temperature.

According to the above fitting results, we can know that the hole concentration of the graded $\mathrm{AlGaN}$ can be improved by decreasing the compensation donors or increasing the acceptors. In our previous study, ${ }^{10}$ we proved that an in-situ $\mathrm{SiN}_{\mathrm{x}}$ mask in N-polar GaN can decrease the concentration of oxygen donors. Therefore, we also inserted a $\mathrm{SiN}_{\mathrm{x}}$ mask in the N-polar GaN template during the growth interruption
TABLE I. The list of hole concentrations for the graded AlGaN with and without the $\mathrm{SiN}_{\mathrm{x}}$ mask depending on different $\mathrm{Cp}_{2} \mathrm{Mg}$ flow rates.

\begin{tabular}{lcc}
\hline \hline $\mathrm{SiN}_{\mathrm{x}}$ mask & $\mathrm{Cp}_{2} \mathrm{Mg}$ flow $(\mathrm{nmol} / \mathrm{min})$ & Hole concentration $\left(\times 10^{17} \mathrm{~cm}^{-3}\right)$ \\
\hline $\mathrm{w} / \mathrm{o}$ & 60 & 6.4 \\
$\mathrm{~W}$ & 50 & 7.0 \\
$\mathrm{~W}$ & 60 & 7.9 \\
$\mathrm{~W}$ & 70 & 9.0 \\
$\mathrm{~W}$ & 80 & 7.9 \\
\hline \hline
\end{tabular}

after the growth of 300-nm-thick GaN. Meanwhile, the growth conditions for the graded $\mathrm{AlGaN}$ on the $\mathrm{GaN}$ template remain unchanged. The hole concentrations measured by Hall measurements at $300 \mathrm{~K}$ are listed in Table I. The result shows that the hole concentration in the graded AlGaN increases from $6.4 \times 10^{17}$ to $7.9 \times 10^{17} \mathrm{~cm}^{-3}$ by inserting the $\mathrm{SiN}_{\mathrm{x}}$ mask. Then, we optimized the flow rate of $\mathrm{Cp}_{2} \mathrm{Mg}$ during the graded $\mathrm{AlGaN}$ growth. We find that the largest hole concentration of $9.0 \times 10^{17} \mathrm{~cm}^{-3}$ is obtained in the graded AlGaN layer when the $\mathrm{Cp}_{2} \mathrm{Mg}$ flow rate is $70 \mathrm{nmol} / \mathrm{min}$.

Furthermore, to confirm the benefits of having the graded AlGaN layer for optical devices, InGaN/GaN multiple-quantum-well (MQW) LEDs with the graded p-AlGaN
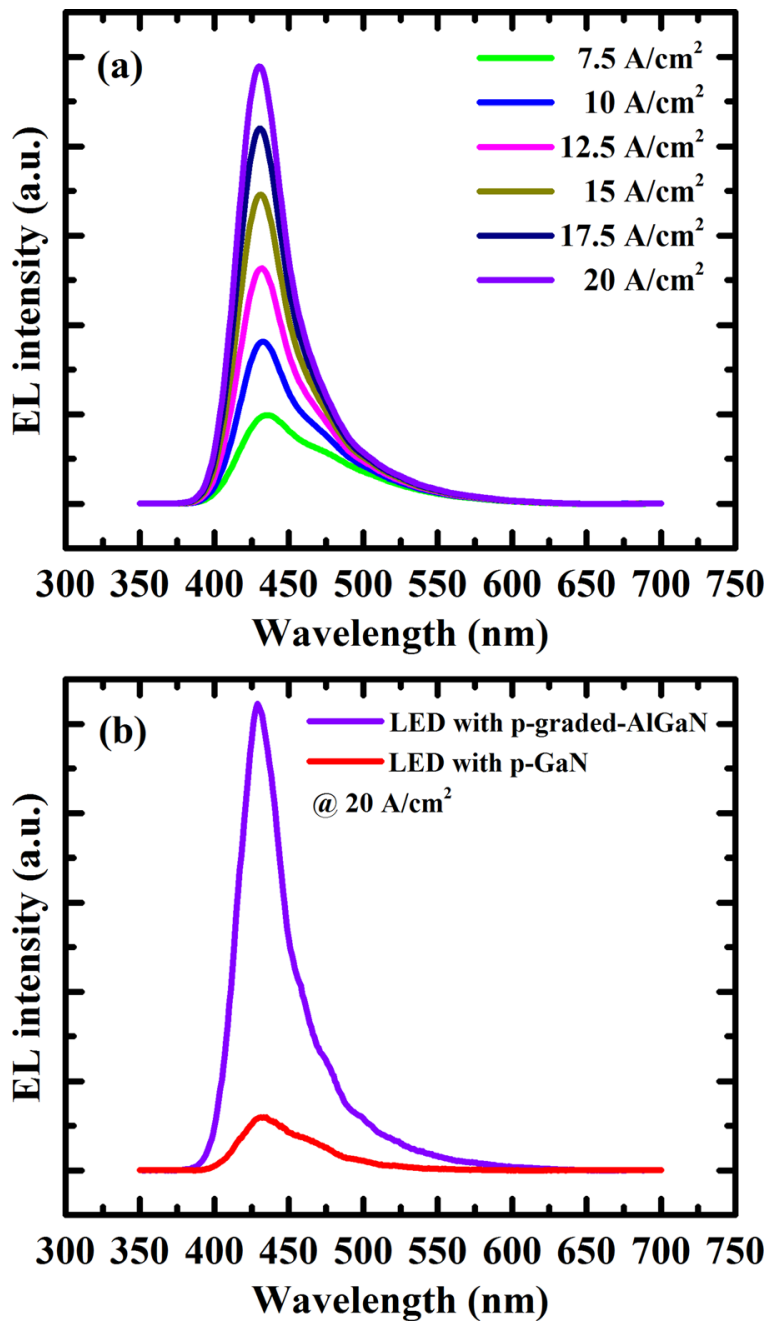

FIG. 3. (a) EL spectra of the LED with graded p-AlGaN at various injection current densities (ranging from $7.5 \mathrm{~A} / \mathrm{cm}^{2}$ to $20 \mathrm{~A} / \mathrm{cm}^{2}$ ). (b) EL spectra of the LEDs with p-GaN and graded p-AlGaN at $20 \mathrm{~A} / \mathrm{cm}^{2}$. 
layer and the $\mathrm{p}-\mathrm{GaN}$ layer were grown by MOCVD, respectively. The epitaxial structure of our LED samples includes a 500 -nm-thick n-type GaN grown on the $2-\mu$ m-thick N-polar GaN template, followed by 5 pairs of MQWs and the graded $\mathrm{p}$-AlGaN layer or the $\mathrm{p}-\mathrm{GaN}$ layer. The growth conditions of the graded $\mathrm{p}-\mathrm{AlGaN}$ layer and the $\mathrm{p}-\mathrm{GaN}$ layer are same as those of the optimized samples mentioned above. The EL spectra of the LED with the graded p-AlGaN layer at different driving current densities (ranging from 7.5 to $20 \mathrm{~A} / \mathrm{cm}^{2}$ ) are shown in Fig. 3(a). The emission peak wavelength shifts from $435 \mathrm{~nm}$ at $7.5 \mathrm{~A} / \mathrm{cm}^{2}$ to $430 \mathrm{~nm}$ at $20 \mathrm{~A} / \mathrm{cm}^{2}$. The slight blue-shift of the emission peak with the increase in injection current can be attributed to the free-carrier screening effect on the polarization field in MQWs. ${ }^{13}$ Figure 3(b) shows the EL spectra of the two LED samples at $20 \mathrm{~A} / \mathrm{cm}^{2}$. We can see that the peak wavelength of the LED with $\mathrm{p}-\mathrm{GaN}$ is the same as that of the LED with graded p-AlGaN. Besides, it can be observed obviously that the EL intensity of the LED with graded p-AlGaN is significantly higher than that of the LED with $\mathrm{p}-\mathrm{GaN}$ due to the higher hole concentration of the graded p-AlGaN.

In conclusion, we demonstrated effective polarizationinduced hole doping via compositionally linearly graded N-polar $\mathrm{Al}_{\mathrm{x}} \mathrm{Ga}_{1-\mathrm{x}} \mathrm{N}(\mathrm{x}=0-0.3)$ grown by MOCVD. The XRD results show that the component and the thickness of the graded AlGaN layer can be accurately controlled by the MOCVD growth process. The absence of the holefreezeout-effect at low temperature indicates that the holes are mainly induced by polarization charges. The increase in the hole concentration at high temperature can be attributed to the thermally activated holes. Besides, we increase the hole concentration of the graded $\mathrm{AlGaN}$ layer from $6.9 \times 10^{17}$ to $9.0 \times 10^{17} \mathrm{~cm}^{-3}$ by optimizing the growth conditions. The stronger EL intensity of the N-polar blue-violet LED with the graded $\mathrm{p}-\mathrm{AlGaN}$, compared to the N-polar
LED with the conventional $\mathrm{p}-\mathrm{GaN}$, indicates the great potential of the polarization-induced doping in N-polar III-nitride light-emitting devices.

This work was supported by the National Key Research and Development Program (No. 2016YFB0400103), the National Natural Science Foundation of China (Nos. 61674068 and 61734001), and the Science and Technology Developing Project of Jilin Province (20150519004JH, 20160101309JC, and 20170204045GX).

${ }^{1}$ A. A. Allerman, M. H. Crawford, A. J. Fischer, K. H. A. Bogart, S. R. Lee, D. M. Follstaedt, P. P. Provencio, and D. D. Koleske, J. Cryst. Growth 272(1-4), 227 (2004).

${ }^{2}$ H. Yoshida, Y. Yamashita, M. Kuwabara, and H. Kan, Appl. Phys. Lett. 93(24), 241106 (2008).

${ }^{3}$ G. Bao, D. Li, X. Sun, M. Jiang, Z. Li, H. Song, H. Jiang, Y. Chen, G. Miao, and Z. Zhang, Opt Express 22(20), 24286 (2014).

${ }^{4}$ J. Li, T. N. Oder, M. L. Nakarmi, J. Y. Lin, and H. X. Jiang, Appl. Phys. Lett. 80(7), 1210 (2002).

${ }^{5}$ J. Simon, V. Protasenko, C. Lian, H. Xing, and D. Jena, Science 327(5961), 60 (2010).

${ }^{6} \mathrm{~S}$. B. Li, T. Zhang, J. Wu, Y. J. Yang, Z. M. Wang, Z. M. Wu, Z. Chen, and Y. D. Jiang, Appl. Phys. Lett. 102(6), 062108 (2013).

${ }^{7}$ L. Zhang, K. Ding, J. C. Yan, J. X. Wang, Y. P. Zeng, T. B. Wei, Y. Y. Li, B. J. Sun, R. F. Duan, and J. M. Li, Appl. Phys. Lett. 97(6), 062103 (2010).

${ }^{8}$ M. Sumiya, K. Yoshimura, K. Ohtsuka, and S. Fuke, Appl. Phys. Lett. 76(15), 2098 (2000).

${ }^{9}$ J. Y. Jiang, Y. T. Zhang, F. Yang, Z. Huang, L. Yan, P. C. Li, C. Chi, D. Q. Zhao, B. L. Zhang, and G. T. Du, Vacuum 119, 63 (2015).

${ }^{10}$ L. Yan, Y. T. Zhang, H. Xu, L. Li, J. Y. Jiang, Z. Huang, X. Han, J. F. Song, and G. T. Du, Mater. Sci. Semicond. Process. 59, 35 (2017).

${ }^{11}$ O. Ambacher, J. Smart, J. R. Shealy, N. G. Weimann, K. Chu, M. Murphy, W. J. Schaff, L. F. Eastman, R. Dimitrov, L. Wittmer, M. Stutzmann, W. Rieger, and J. Hilsenbeck, J. Appl. Phys. 85(6), 3222 (1999).

${ }^{12}$ H. Okumura, D. Martin, M. Malinverni, and N. Grandjean, Appl. Phys. Lett. 108(7), 072102 (2016).

${ }^{13}$ F. Della Sala, A. Di Carlo, P. Lugli, F. Bernardini, V. Fiorentini, R. Scholz, and J. M. Jancu, Appl. Phys. Lett. 74(14), 2002 (1999). 